\title{
Análise da presença de síndrome metabólica em idosos atendidos no Projeto Atendimento Multidisciplinar ao Idoso (AMI) em Campo Grande, MS
}

\section{Analysis of metabolic syndrome presence in the elderly attended by the Project Multidisciplinary Care for the Elderly (AMI) in Campo Grande, MS}

Análisis de la presencia de síndrome metabólico en ancianos atendidos en el Proyecto Atendimiento Multidisciplinar al Anciano (AMI) en Campo Grande, MS

Thaís de Sousa da Silva ${ }^{1}$ Camila Nunes de Souza ${ }^{2}$ Cláudia Gonçalves Gouveia ${ }^{3}$ Luciane Perez da Costa ${ }^{4}$

${ }^{1}$ Especialista pelo Programa de Residência Multiprofissional em Cuidados Continuados Integrados - Atenção à Saúde do Idoso da Universidade Federal de Mato Grosso do Sul (UFMS). Graduada em Nutrição pela Universidade Anhanguera-Uniderp.E-mail: sousa.thais@outlook.com, Orcid: http://orcid.org/0000-0002-1189-8229.

${ }^{2}$ Especialista pelo Programa de Residência Multiprofissional em Atenção ao Paciente Crítico da Universidade Federal de Mato Grosso do Sul (UFMS).

Graduada em Nutrição pela Universidade Anhanguera-Uniderp. E-mail: nutricamilanunes@hotmail.com, Orcid: http://orcid.org/0000-0002-9392-1401

${ }^{3}$ Biomédica do Hospital São Julião. E-mail: claudia@saojuliao.org.br, Orcid: http://orcid.org/0000-0003-3810-2734

${ }^{4}$ Doutoranda e mestre em Biotecnologia pela Universidade Católica Dom Bosco (UCDB). Especialista em Nutrição Clínica pela Universidade AnhangueraUniderp. Graduada em Nutrição pela UCDB. E-mail: perezlu10@hotmail.com, Orcid: http://orcid.org/0000-0002-3524-588X 
Resumo: A Síndrome Metabólica (SM) é constituída por vários fatores que compõem os critérios diagnósticos. Quanto mais fatores associados, maiores os riscos do desenvolvimento da SM e de doenças cardiovasculares. Hábito alimentar inadequado associado ao declínio da atividade física dos indivíduos contribui para o aumento da prevalência de obesidade, que também é considerada como um dos componentes da SM. O objetivo deste trabalho foi analisar a presença de Síndrome Metabólica em idosos atendidos no Projeto Atendimento Multidisciplinar ao Idoso (AMI) de Campo Grande, MS, conforme os critérios diagnósticos NCEP-ATP III, IDF e JIS. Trata-se de um estudo quantitativo, descritivo, transversal e retrospectivo, através de análise do prontuário. A prevalência de Síndrome Metabólica nos idosos participantes da pesquisa foi de $47,22 \%$ para os critérios NCEP-ATP III e IDF, e $50 \%$ para o critério JIS, mostrando-se mais sensível ao critério JIS.

Palavras-chave: síndrome metabólica; doenças cardiovasculares; saúde do idoso.

\begin{abstract}
The Metabolic Syndrome (MS) consists of several factors that make up the diagnostic criteria. The more associated factors, the greater the risks of developing MS and cardiovascular diseases. Inadequate eating habits associated with the decline in physical activity of individuals contribute to the increased prevalence of obesity, which is also considered as one of the components of MS. The objective of this study was to analyze the presence of Metabolic Syndrome in the elderly served in the Project Multidisciplinary Care for the Elderly (AMI) in Campo Grande, MS, according to the NCEP-ATP III, IDF and JIS diagnostic criteria. This is a quantitative, descriptive, cross-sectional and retrospective study through analysis of medical records.. The prevalence of Metabolic Syndrome in the elderly participants of the study was $47.22 \%$ for the NCEP-ATP III and IDF criteria, and $50 \%$ for the JIS criterion, being more sensitive to the JIS criterion.
\end{abstract}

Keywords: metabolic syndrome; cardiovascular diseases; health of the elderly.

Resumen: El Síndrome Metabólico (SM) está constituido por varios factores que componen los criterios diagnósticos. Cuantos más factores asociados, mayores son los riesgos del desarrollo de la SM y de enfermedades cardiovasculares. El hábito alimentario inadecuado asociado al declive de la actividad física de los individuos contribuye al aumento de la prevalencia de obesidad, que también se considera como uno de los componentes de SM. El objetivo de este trabajo fue analizar la presencia de Síndrome Metabólico en ancianos atendidos en el Proyecto Atendimiento Multidisciplinar al Anciano (AMI) de Campo Grande, MS, conforme a los criterios diagnósticos NCEP-ATP III, IDF y JIS. Se trata de un estudio cuantitativo, descriptivo, transversal y retrospectivo, a través del análisis del prontuario. La prevalencia de Síndrome Metabólico en los ancianos participantes en la investigación fue del $47,22 \%$ para los criterios NCEP-ATP III e IDF, y 50\% para el criterio JIS, mostrándose más sensible al criterio JIS.

Palabras clave: síndrome metabólico; enfermedades cardiovasculares; salud del anciano. 


\section{INTRODUÇÃO}

O envelhecimento pode ser caracterizado como um processo natural que se desenvolve ao longo da vida, mudando de forma progressiva as funções fisiológicas e psicossociais do indivíduo. Segundo a Organização Mundial de Saúde (OMS), a velhice inicia-se aos 60 anos em países em desenvolvimento e aos 65 anos em países desenvolvidos, sendo que a situação socioeconômica de cada país é um critério a se considerar.

O aumento da expectativa de vida não é sinônimo, hoje, de envelhecer com saúde, pois o processo de envelhecimento traz consigo doenças próprias da idade, que surgem devido a alterações fisiológicas, mudanças na composição corporal e nos hábitos alimentares, afetando também a qualidade de vida desses indivíduos. À medida que a idade chega, os números de doenças crônicas não transmissíveis aumentam, com destaque para as doenças cardiovasculares (DCV), que estão entre as principais causas de morbimortalidade desta população.

A Síndrome Metabólica (SM), uma das doenças que mais acometem os idosos, é caracterizada como um distúrbio complexo de alterações fisiopatológicas, associada a fatores de risco relacionados ao perfil lipídico (dislipidemia) e glicêmico (anormalidade da glicemia de jejum, tolerância diminuída à glicose ou presença de diabetes mellitus, DM), níveis pressóricos (hipertensão arterial sistêmica) e obesidade abdominal.

Entre os vários critérios diagnósticos existentes para a SM, os mais utilizados são: National Cholesterol Education Program's Adult Treatment Panel III (NCEP-ATP III), que classifica a síndrome pela presença de pelo menos três critérios (podendo ser glicemia de jejum $\geq 100$ mg/dL; circunferência abdominal $(C A) \geq 102 \mathrm{~cm}$ para homens ou $\geq 88 \mathrm{~cm}$ para mulheres; $\mathrm{HDL}<40$ $\mathrm{mg} / \mathrm{dL}$ para homens ou $<50 \mathrm{mg} / \mathrm{dL}$ para mulheres; triglicerídeos $\geq 150 \mathrm{mg}$ / dL; e/ou pressão arterial $\geq 130 / 85$ mmHg); International Diabetes Federation (IDF), que prevê a presença obrigatória do critério de circunferência abdominal, sendo $\geq 94 \mathrm{~cm}$ para homens ou $\geq 80 \mathrm{~cm}$ para mulheres, associada a dois outros fatores listados no NCEP-ATP III; e Joint Interim Statement (JIS), que classifica a presença de pelo menos três dos critérios citados no NCEP- 
ATP III, porém o valor de corte para a circunferência abdominal é $\geq 94 \mathrm{~cm}$ para homens ou $\geq 80 \mathrm{~cm}$ para mulheres.

Diante do processo de envelhecimento, nota-se a necessidade de serviços e programas de saúde voltados para a população idosa. Desta maneira, surge o Atendimento Multidisciplinar ao Idoso (AMI), um projeto social e de pesquisa desenvolvido por uma equipe de profissionais como nutricionistas, médicos, enfermeiros, psicólogos e fisioterapeutas. O AMI busca estabelecer medidas de caráter preventivo, terapêutico e reabilitador, visando à prevenção de doenças e à promoção de saúde através de palestras, oficinas e atividades educativas e culturais, bem como promovendo a socialização entre os idosos.

O objetivo deste trabalho é identificar a presença de Síndrome Metabólica em idosos atendidos no AMI em Campo Grande, MS, conforme os critérios diagnósticos: NCEP-ATP III, IDF e JIS.

\section{MÉTODO}

Esta pesquisa teve uma abordagem quantitativa, descritiva e transversal, sendo aprovada pelo Comitê de Ética em Pesquisa em Seres Humanos da Universidade Federal de Mato Grosso do Sul (CEP/UFMS), parecer n. 2.407.475 (CAEE 79997017.8.0000.0021). A partir da aprovação, deu-se início à coleta de dados, a qual foi realizada nos meses de abril a julho de 2018, no ambulatório de um hospital de retaguarda na cidade de Campo Grande, Mato Grosso do Sul.

Para a coleta dos dados, foi utilizado um formulário de pesquisa desenvolvido e padronizado pelas pesquisadoras (Apêndice 1). Este foi preenchido conforme a coleta dos seguintes dados: identificação, gênero, idade, se o indivíduo é etilista e/ou tabagista, se realiza a prática de alguma atividade física, se realiza controle glicêmico e/ou pressórico, diagnósticos clínicos (como diabetes mellitus, hipertensão arterial sistêmica, dislipidemias, entre outras comorbidades), frequência alimentar, antropometria (peso, altura, IMC e circunferência abdominal) e exames laboratoriais (dosagem de glicemia de jejum, colesterol total, HDL, LDL e triglicerídeos).

Lipschitz (1994) classifica o IMC para idosos conforme a tabela abaixo (Tabela 1). 
Tabela 1- Classificação IMC para idosos

\begin{tabular}{cc}
\hline CLASSIFICAÇÃO & IMC \\
\hline Baixo peso & $<22 \mathrm{~kg} / \mathrm{m}^{2}$ \\
Eutrofia & $22-27 \mathrm{~kg} / \mathrm{m}^{2}$ \\
Sobrepeso & $>27 \mathrm{~kg} / \mathrm{m}^{2}$ \\
\hline
\end{tabular}

Fonte: Lipschitz (1994).

Quanto à circunferência abdominal, a OMS (1995) classifica o sexo feminino e masculino de acordo com a circunferência com risco aumentado ou muito aumentado para desenvolver complicações metabólicas (Tabela 2).

Tabela 2-Classificação de circunferência abdominal e risco para desenvolver complicações metabólicas

\begin{tabular}{ccc} 
& Risco aumentado & Risco muito aumentado \\
\hline Feminino & $\geq 80 \mathrm{~cm}$ & $\geq 88 \mathrm{~cm}$ \\
Masculino & $\geq 94 \mathrm{~cm}$ & $\geq 102 \mathrm{~cm}$ \\
\hline
\end{tabular}

Fonte: OMS (1995).

Os dados foram extraídos do prontuário de idosos participantes do Projeto AMI que estavam dentro dos critérios de inclusão: prontuários de indivíduos participantes do Projeto AMI, ter 60 anos ou mais e ter passado pela consulta com o setor de Nutrição no período de janeiro de 2017 a março de 2018. Foram excluídos da pesquisa prontuários de indivíduos com idade igual ou inferior a 59 anos, indígenas e os privados de liberdade, indivíduos que não deambulam (cadeirantes e acamados) e indivíduos que se alimentam por via alternativa (nutrição enteral) exclusiva e/ou associada à via oral.

Os prontuários foram avaliados individualmente, identificando os indivíduos com presença de Síndrome Metabólica e sem presença de Síndrome Metabólica, conforme as variáveis estabelecidas nos critérios diagnósticos NCEP-ATP III, IDF e JIS (Tabela 3), apresentando a frequência de Síndrome Metabólica no grupo estudado. 
Tabela 3 - Critérios NCEP-ATP III, IDF e JIS para diagnóstico de Síndrome Metabólica

\begin{tabular}{|c|c|c|c|}
\hline & NCEP-ATP III & IDF & JIS \\
\hline Pressão arterial & $\geq 130 / 85 \mathrm{mmHg}$ & $\begin{array}{l}\geq 130 / 85 \mathrm{mmHg} \text { ou } \\
\text { tratamento de HAS }\end{array}$ & $\begin{array}{l}\geq 130 / 85 \mathrm{mmHg} \text { ou } \\
\text { tratamento de HAS }\end{array}$ \\
\hline Circunferência & $\hat{o} \geq 102 \mathrm{~cm}$ & $\widehat{o} \geq 94 \mathrm{~cm}$ & $\widehat{\sigma} \geq 94 \mathrm{~cm}$ \\
\hline abdominal & $q \geq 88 \mathrm{~cm}$ & $q \geq 80 \mathrm{~cm}$ & $q \geq 80 \mathrm{~cm}$ \\
\hline Glicose & $\geq 110 \mathrm{mg} / \mathrm{dL}$ & $\geq 110 \mathrm{mg} / \mathrm{dL}$ & $\geq 110 \mathrm{mg} / \mathrm{dL}$ \\
\hline Triglicerídeos & $\geq 150 \mathrm{mg} / \mathrm{dL}$ & $\geq 150 \mathrm{mg} / \mathrm{dL}$ & $\geq 150 \mathrm{mg} / \mathrm{dL}$ \\
\hline HDL & $\begin{array}{l}\widehat{\jmath}<40 \mathrm{mg} / \mathrm{dL} \\
q<50 \mathrm{mg} / \mathrm{dL}\end{array}$ & 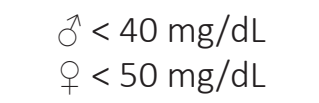 & $\begin{array}{l}\hat{\jmath}<40 \mathrm{mg} / \mathrm{dL} \\
q<50 \mathrm{mg} / \mathrm{dL}\end{array}$ \\
\hline Critérios SM & $\begin{array}{l}\text { Três ou mais } \\
\text { componentes }\end{array}$ & $\begin{array}{c}\text { CA mais } 2 \\
\text { componentes }\end{array}$ & $\begin{array}{l}\text { Três ou mais } \\
\text { componentes }\end{array}$ \\
\hline
\end{tabular}

Fonte: Saad et al. (2013).

NCEP-ATP III: National Cholesterol Education Program's Adult Treatment Panel III; IDF: International Diabetes Federation; JIS: Joint Interim Statement; HAS: Hipertensão Arterial Sistêmica; HDL: high-density lipoprotein; $\widehat{o}$ : masculino; $\bigcirc$ : feminino.

A pesquisa considerou os critérios National Cholesterol Education Program's Adult Treatment Panel III (NCEP-ATP III), International Diabetes Federation (IDF) e Joint Interim Statement (JIS) por serem os mais utilizados, de acordo com a literatura.

Os dados obtidos foram organizados e analisados através da planilha Microsoft Excel ${ }^{\circledR}$ e a avaliação estatística foi realizada pelo software SPSS (Statistical Package for the Social Sciences) versão 18.0, através da análise descritiva dos resultados por meio da média \pm desvio padrão (DP) para as variáveis contínuas. As variáveis estudadas foram testadas em relação à sua normalidade para escolha do(s) teste(s) estatístico(s) mais apropriado(s), considerando um nível de $5 \%$ de significância $(p<0,05)$.

Os resultados dos exames laboratoriais foram obtidos por meio dos dados contidos nos prontuários e no sistema informatizado do hospital, sendo que os valores de referência são determinados e seguem a padronização do laboratório da instituição. 


\section{RESULTADOS}

Foram investigados 36 prontuários, sendo $61 \%$ do sexo feminino e $39 \%$ do sexo masculino. Os participantes apresentaram idade média de $66,95 \pm 21,03$ (61-89) anos.

Sobre as condições clínicas observadas, 66,67\% dos idosos apresentaram hipertensão arterial sistêmica; 44,44\%, depressão, hipotireoidismo, ansiedade e osteoporose; $27,78 \%$, diabetes mellitus; 16,67\%, cardiopatia e dislipidemia; e 13,89\%, hipertrigliceridemia e hipercolesterolemia. Quanto ao tratamento medicamentoso das comorbidades, $83 \%$ realizam tratamento medicamentoso, enquanto $17 \%$ não realizam e/ou não precisam tratar suas comorbidades através de medicamentos.

Sobre os componentes da Síndrome Metabólica (Tabela 4), a média de peso da população estudada foi de 72,37 kg $\pm 13,26$ ( $p=0,0093$ ) e IMC $28,78 \mathrm{~kg} / \mathrm{m}^{2} \pm 6,08(p=0,0046)$. As médias do IMC entre os idosos, separados por sexo, demonstraram que, segundo a classificação de Lipschitz (1994), o sexo feminino apresentou sobrepeso $(30,32 \pm 6,623)$ e o sexo masculino, eutrofia $(26,38 \pm 4,296)$, apontando diferença estatística significativa $(p=0,0046)$. Ambos os sexos apresentaram risco elevado para o aparecimento de doenças cardiovasculares através da circunferência abdominal, segundo a recomendação da OMS (1995), não havendo diferença estatística.

Observou-se, também, que a média de glicose em jejum apresentou-se dentro dos padrões de normalidade para o sexo feminino $(107,95 \pm$ $14,873)$ e aumentada para o sexo masculino $(114,66 \pm 23,186)$, segundo os padrões referenciados pela Sociedade Brasileira de Diabetes (2002), em que o limite para alterações glicêmicas é de até $110 \mathrm{mg} / \mathrm{dL}$ para ambos os sexos, apontando diferença estatística significativa $(p=0,0068)$. Quanto ao perfil lipídico, os triglicerídeos e o HDL apresentaram-se dentro dos valores de normalidade segundo os padrões estipulados pela III Diretrizes Brasileiras sobre Dislipidemias (2002). 
Tabela 4- Componentes da Síndrome Metabólica de acordo com o sexo em uma unidade ambulatorial em Campo Grande, MS, 2018

\begin{tabular}{|c|c|c|c|}
\hline & $\begin{array}{c}\text { Feminino }(n=22) \\
\text { Média } \pm \text { DP }\end{array}$ & $\begin{array}{c}\text { Masculino }(n=14) \\
\text { Média } \pm \text { DP }\end{array}$ & Valor de $\mathbf{P}$ \\
\hline IMC $\left(\mathrm{kg} / \mathrm{m}^{2}\right)$ & $30,32 \pm 6,623$ & $26,38 \pm 4,296$ & 0,0046 \\
\hline $\begin{array}{l}\text { Circunferência } \\
\text { abdominal }(\mathrm{cm})\end{array}$ & $98,48 \pm 11,77$ & $98,07 \pm 11,64$ & $>0,10$ \\
\hline Glicemia (mg/dl) & $107,95 \pm 14,873$ & $114,66 \pm 23,186$ & 0,0068 \\
\hline $\begin{array}{l}\text { Triglicerídeos } \\
\text { (mg/dl) }\end{array}$ & $126,42 \pm 73,23$ & $105,18 \pm 43,499$ & 0,0329 \\
\hline $\begin{array}{c}\text { HDL - Colesterol } \\
(\mathrm{mg} / \mathrm{dl})\end{array}$ & $53,80 \pm 17,515$ & $48,86 \pm 13,201$ & 0,007 \\
\hline
\end{tabular}

Fonte: Pesquisa de campo.

Sabe-se que fatores externos, como hábitos alimentares inadequados, tabagismo, etilismo e inatividade física, influenciam no aparecimento da SM. Sendo assim, foi investigada a prática de atividade física e os hábitos alimentares. Quanto à prática de atividade física, apenas 17 indivíduos realizam algum tipo de atividade, como caminhada, alongamento, ginástica, aeróbico e hidroginástica. As mulheres destacaram-se nesse quesito, representando $76,50 \%$, enquanto os homens somaram apenas $23,50 \%$ (Gráfico 1). 
Gráfico 1- Número de praticantes de atividade física separados por sexo em uma unidade ambulatorial em Campo Grande, MS, 2018

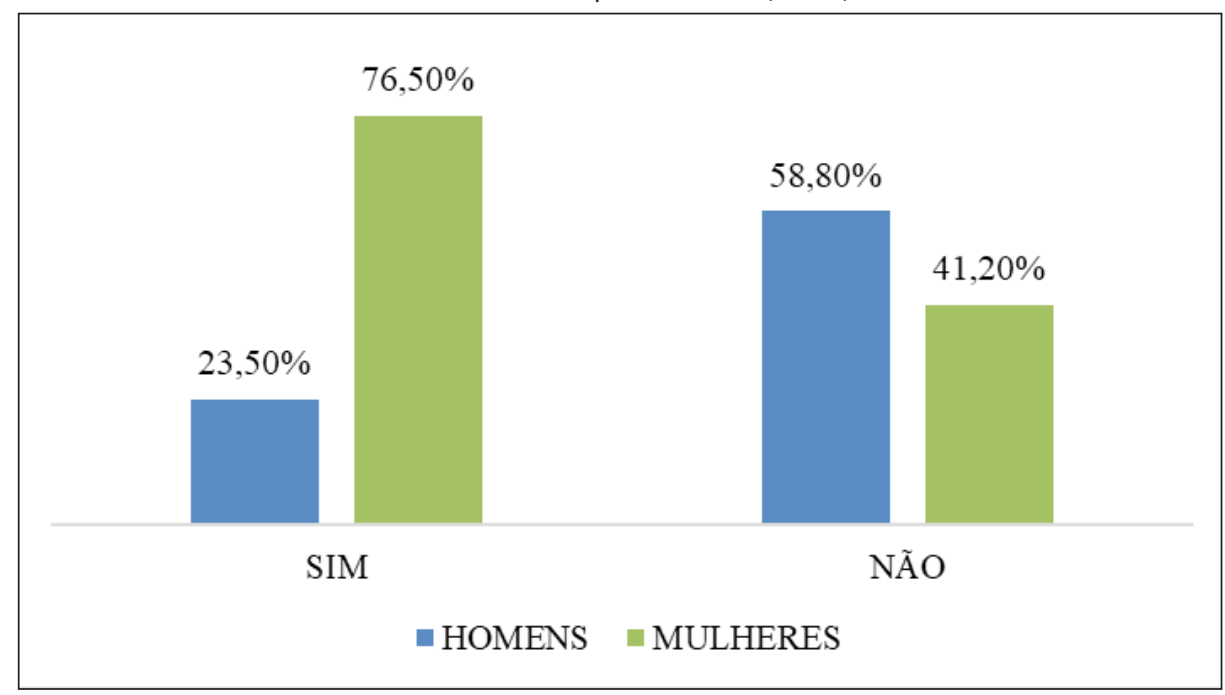

Fonte: Pesquisa de campo.

Quanto aos hábitos alimentares, foi analisada a quantidade de refeições realizadas durante o dia, sendo que 30,36\% fazem 3 refeições ou menos ao dia, 25\% fazem 4 e 5 refeições ao dia, respectivamente, e apenas 11,11\% realizam 6 refeições ou mais ao dia.

Para avaliar a presença de SM, este estudo utilizou os critérios propostos pelo NCEP-ATP III, IDF e JIS, sendo classificados com a síndrome, respectivamente, $47,22 \%, 47,22 \%$ e 50,00\% dos indivíduos participantes (Gráfico 2). 
Gráfico 2- Frequência da presença de Síndrome Metabólica na população estudada, de acordo com os critérios NCEP-ATP III, IDF e JIS, em uma unidade ambulatorial em Campo Grande, MS, 2018

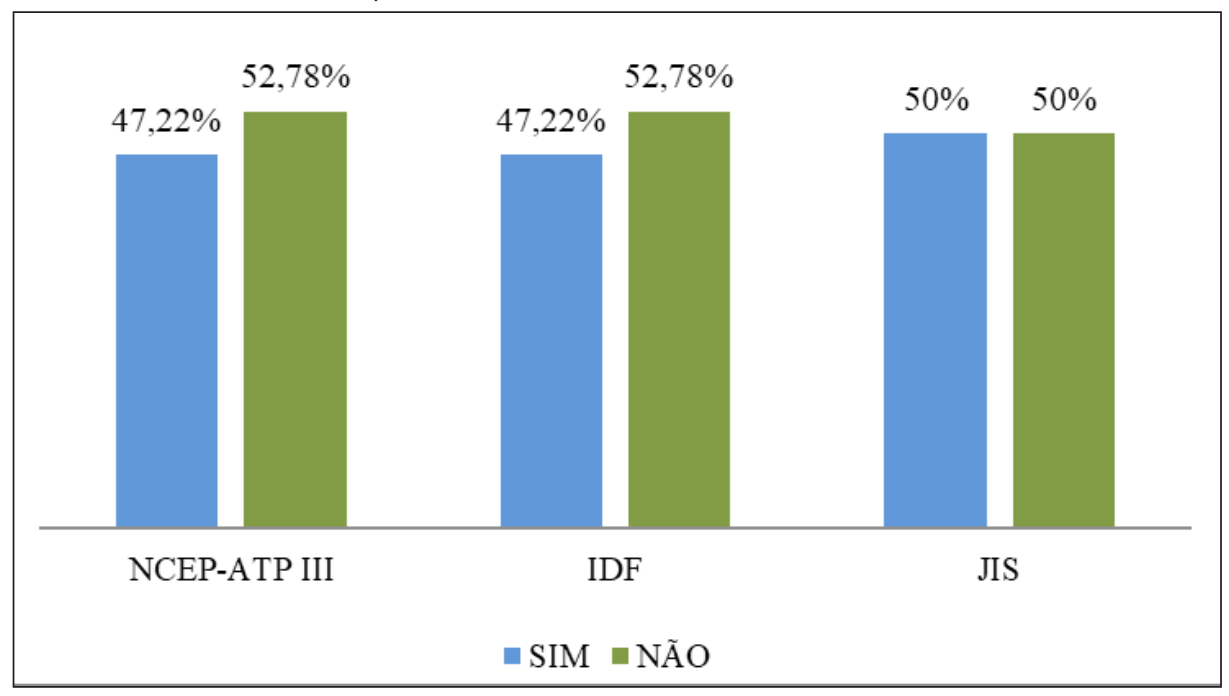

Fonte: Pesquisa de campo.

NCEP-ATP III: Third Report of the National Cholesterol Education Program; IDF: International Diabetes Federation; JIS: Joint Interim Statement.

A SM apresentou-se mais frequente no sexo feminino nos três critérios avaliados, correspondendo a 58,80\% segundo os critérios NCEP-ATP III e IDF, e 55,60\% pelo critério JIS. A população masculina representou 41,20\%, $41,20 \%$ e 44,40\% pelos critérios NCEP-ATP III, IDF e JIS, respectivamente.

\section{DISCUSSÃO}

A Síndrome Metabólica está relacionada diretamente às doenças crônicas não transmissíveis e aos componentes essenciais que a caracterizam, como obesidade abdominal, alterações glicêmicas, hipertensão arterial sistêmica e dislipidemia, além de sofrer influência de fatores externos, como hábitos alimentares inadequados, tabagismo, etilismo e inatividade física. Quanto maior a prevalência desses componentes no indivíduo, maiores são as chances de desenvolver comorbidades como diabetes mellitus, doenças 
cardiovasculares, aterosclerose, esteatose hepática, entre outras (ROCHA; MELO; MENEZES, 2016).

O diagnóstico da Síndrome Metabólica em idosos vem sendo muito estudado (BOLZAN; MACHADO; SCHUCH, 2017). No presente estudo, avaliou-se a presença de Síndrome Metabólica de acordo com os critérios NCEP-ATP III, IDF e JIS e foi possível verificar que os três foram sensíveis ao público estudado, destacando-se o critério JIS, que classificou $50 \%$ dos idosos. O estudo realizado por Saad et al. (2013), com uma população de idosos em uma unidade ambulatorial no Rio de Janeiro, mostrou o critério JIS como o mais sensível, classificando $69,1 \%$ dos idosos. A presença dos fatores de risco nos idosos e, consequentemente, o desenvolvimento da síndrome já são esperados em decorrência das alterações metabólicas e fisiológicas consequentes do processo de envelhecimento (VITOLO, 2015).

Neste estudo, foi possível verificar que o público feminino foi o mais predominante na população avaliada, sendo o mais participativo e com maior número de diagnósticos de Síndrome Metabólica. No estudo de Zoraski et al. (2017), realizado em idosos de Nova Roma, Rio Grande do Sul, destacou-se maior participação do sexo feminino, correspondendo a 56\% da população estudada. Ibiapina et al. (2015) realizaram um estudo com idosos que frequentavam um centro de convivência no município de Teresina, Piauí, e observaram que o sexo feminino também era o mais participativo e com o maior número de diagnósticos de Síndrome Metabólica. Tavares et al. (2018) afirmam que o sexo feminino é mais susceptível ao aumento da circunferência abdominal e ao risco para doenças cardiovasculares devido às alterações hormonais que ocorrem na menopausa. Segundo alguns estudos, as mulheres são as que mais buscam e se utilizam dos serviços de saúde, seja porque se preocupam mais com a saúde, seja porque têm mais iniciativa e facilidade de acesso a esses serviços (SANTOS; FERREIRA; MORI, 2017).

Em relação aos componentes da SM, a avaliação do estado nutricional é capaz de diagnosticar alterações metabólicas, permitindo avaliar o grau de risco do indivíduo para o desenvolvimento de doenças crônicas não transmissíveis. Segundo o estudo realizado por Vieira, Peixoto e Silveira (2014) com idosos usuários do Sistema Único de Saúde (SUS), as médias do IMC 
entre os idosos, separados por sexo, demonstram que o sexo masculino se destacou com maiores valores quanto ao IMC de sobrepeso, CA aumentada e glicemia alterada, resultados que corroboram os dados levantados.

A circunferência abdominal também é um dos fatores que mais se relacionam (NASCIMENTO et al., 2017) com o desenvolvimento de doenças crônicas (MOTA et al., 2011). Assim, o estudo de Barroso et al. (2017) afirma que a obesidade central está associada a doenças como diabetes, dislipidemias e hipertensão arterial sistêmica, contribuindo fortemente para complicações metabólicas e outros agravos à saúde.

Além disso, outros dois fatores importantes, capazes de colaborar para o desenvolvimento de doenças crônicas, são a falta de atividade física e a presença de comorbidades. No estudo realizado por Calixto et al. (2016), 67\% do público avaliado era sedentário e apresentou hipertensão arterial sistêmica como a comorbidade mais prevalente.

Com o aumento da frequência de SM na população idosa, aumenta também o número de casos de comorbidades como doenças cardiovasculares. Esse cenário reflete diretamente na saúde pública e exige que mudanças na assistência à saúde sejam realizadas através da criação de programas e projetos educacionais, os quais devem estabelecer atividades que promovam transformações no estilo de vida, em busca de uma qualidade de vida melhor (FORD; GILES; MOKDAD, 2004).

Entre as mudanças no estilo de vida, pode-se destacar os hábitos alimentares. Uma dieta balanceada, fracionada em pequenas porções, rica em nutrientes, é o ponto-chave para evitar o ganho de peso em excesso e, consequentemente, o aumento da circunferência abdominal, evitando, assim, o desencadeamento da SM (PINTO-E-SILVA et al., 2016).

Contudo mudanças nesses fatores atuam como medidas preventivas que devem ser adotadas a fim de diminuir a prevalência desse agravo e minimizar os impactos na saúde dos idosos. Destaca-se como ação a identificação precoce dos principais fatores de risco que causam morbidade nesse público, além do controle e acompanhamento efetivo destes em nível de atenção básica, objetivando promover um controle mais eficiente da síndrome (SCHULTZ; WICHMANN; COUTO, 2014). 
Portanto estes resultados tratam de dados preocupantes, devido à associação da Síndrome Metabólica com o aparecimento das doenças crônicas e por ela estar associada ao aumento da morbimortalidade dessa população (BORTOLETTO et al., 2016).

\section{CONCLUSÃO}

Nesta pesquisa, foi possível verificar que a frequência de SM nos idosos participantes foi demonstrada nos três critérios diagnósticos, mostrando-se mais sensível ao critério JIS. Dos indivíduos avaliados, o sexo feminino destacou-se com maior participação no projeto e na prática de atividade física e apresentou maior frequência quanto à classificação de SM, nos três critérios avaliados.

Os resultados apresentados através desta pesquisa servirão para uma melhor avaliação desse público, tanto da parte da Nutrição quanto da equipe multiprofissional, visando a uma sistematização do atendimento, desde a escolha do melhor critério diagnóstico até o desenvolvimento de medidas preventivas e terapêuticas, para promover mudanças de comportamento e melhor qualidade de vida.

Assim, é importante que a população idosa seja incentivada a realizar mudanças no estilo de vida quanto à adoção de hábitos alimentares mais saudáveis, associados à prática de atividade física, visando intensificar estratégias de prevenção e promoção à saúde, além de estabelecer medidas preventivas, terapêuticas e reabilitadoras.

\section{REFERÊNCIAS}

BARROSO, T. A. et al. Associação entre a obesidade central e a incidência de doenças e fatores de risco cardiovascular. International Journal of Cardiovascular Sciences, Rio de Janeiro, v. 30, n. 5, p. 416-24, set./out. 2017.

BOLZAN, A. G.; MACHADO, L.; SCHUCH, N. J. Caracterização dos componentes da síndrome metabólica em idosas segundo dois critérios diagnósticos. Disciplinarum Scientia. Série: Ciências da Saúde, Santa Maria, RS, v. 18, n. 3, p. 585-92, 2017. 
BORTOLETTO, M. S. S. et al. Síndrome metabólica, componentes e fatores associados em adultos de 40 anos ou mais de um município da Região Sul do Brasil. Cadernos Saúde Coletiva, Rio de Janeiro, v. 24, n. 1, p. 32-40, 2016.

CALIXTO, S. C. S. et al. Prevalência da síndrome metabólica em idosos. Revista Saúde em Foco, Teresina, PI, v. 3, n. 2, p. 119-35, jul./dez. 2016.

FORD, E. S.; GILES, W. H.; MOKDAD, A. H. Increasing prevalence of the metabolic syndrome among US adults. Diabetes Care, v. 27, n. 10, p. 2444-9, out. 2004.

IBIAPINA, D. F. N. et al. Prevalência da síndrome metabólica em idosos. Revista de enfermagem UFPE [on-line], Recife, v. 9, supl. 9, p. 9964-70, nov. 2015.

LIPSCHITZ, D. A. Screening for nutritional status in the elderly. Primary Care, v. 21, n. 1, p. 55-67, 1994.

MOTA, J. F. et al. Indicadores antropométricos como marcadores de risco para anormalidades metabólicas. Ciência \& Saúde Coletiva, Rio de Janeiro, v. 16, n. 9, p. 3901-8, 2011.

NASCIMENTO, M. M. et al. Comparação e concordância de critérios à classificação do IMC de idosas fisicamente ativas, residentes no Sertão Nordestino. Journal of Human Growth and Development, São Paulo, v. 27, n. 3, p. 342-49, set./dez. 2017.

ORGANIZAÇÃO MUNDIAL DE SAÚDE. Physical status: the use and interpretation of anthropometry. Geneva: OMS, 1995.

PINTO-E-SILVA, M. E. M. et al. Alimentação saudável: prevenindo a síndrome metabólica. Segurança Alimentar e Nutricional, Campinas, SP, v. 23, n. 2, p. 94454, 2016.

ROCHA, F. L.; MELO, R. L. P.; MENEZES, T. N. Fatores associados à síndrome metabólica em idosos do interior do Nordeste brasileiro. Revista Brasileira de Geriatria e Gerontologia, Rio de Janeiro, v. 19, n. 6, p. 978-86, nov./dez. 2016.

SAAD, M. A. N. et al. Prevalência de Síndrome Metabólica em idosos e concordância entre quatro critérios diagnósticos. Arquivos Brasileiros de Cardiologia, Rio de Janeiro, v. 102, n. 3, p. 263-9, 2013.

SANTOS, P. C. M.; FERREIRA, A. L. L.; MORI, R. M. S. C. Frequência da síndrome metabólica em idosos cadastrados no Programa Saúde do Idoso de uma unidade 
municipal de saúde de Belém - PA. RASBRAN - Revista da Associação Brasileira de Nutrição [on-line], São Paulo, ano 8, n. 1, p. 75-81, jan./jun. 2017.

SCHULTZ, R. D. P.; WICHMANN, F. A.; COUTO, A. N. Adesão e eficácia do aconselhamento dietético após intervenção nutricional em mulheres com fatores de risco para a síndrome metabólica. Revista Cinergis, Santa Cruz do Sul, RS, v. 15, n. 3, p. 123-8, 2014.

TAVARES, D. S. et al. Perfil de idosos com síndrome metabólica e fatores associados às possíveis interações medicamentosas. Revista Brasileira de Geriatria e Gerontologia, Rio de Janeiro, v. 21, n. 2, p. 168-79, abr./mar. 2018.

VIEIRA, E. C.; PEIXOTO, M. R. G.; SILVEIRA, E. A. Prevalência e fatores associados à Síndrome Metabólica em idosos usuários do Sistema Único de Saúde. Revista Brasileira de Epidemiologia, São Paulo, v. 17, n. 4, p. 805-17, out./dez. 2014.

VITOLO, M. R. Nutrição: da gestação ao envelhecimento. 2. ed. Rio de Janeiro: Rúbio, 2015.

ZORASKI, H. et al. Síndrome metabólica em idosos de Nova Roma do Sul, RS: prevalência e fatores associados. ABCS Health Sciences, Santo André, SP, v. 42, n. 3, p. 147-55, 2017. 


\section{APÊNDICE A - FORMULÁRIO DE PESQUISA}

\section{FORMULÁRIO DE PESQUISA}

IDENT.:

GÊNERO: F( ) M( )

IDADE: ANOS

TABAGISTA: NÃO ( ) SIM ( ) FREQ.: CIGARROS/DIA ETILISTA NÃO ( ) SIM ( ) FREQ.:

REALIZA ATIVIDADE FÍSICA? NÃO（） SIM（）, QUAL?

REALIZA CONTROLE GLICÊMICO? NÃo ( ) SIM ( ), REGULARIDADE: ( ) DIÁRIO ( ) SEMANAL ( ) MENSAL ( ) ANUAL REALIZA CONTROLE PRESSÓRICO? NÃO ( ) SIM ( ), REGULARIDADE: ( ) DIÁRIO ( ) SEMANAL ( ) MENSAL ( ) ANUAL

INQUÉRITO ALIMENTAR

REFEIÇÕES REALIZADAS POR DIA: DUAS（） TRÊS（） QUATRO（） CINCO（） SEIS OU MAIS（）

\section{QUESTIONÁRIO DE FREQUÊNCIA ALIMENTAR - GRUPOS ALIMENTARES}

\begin{tabular}{|l|l|l|l|l|l|}
\hline & DIARIAMENTE & SEMANALMENTE & MENSALMENTE & EVENTUALMENTE & NUNCA \\
\hline $\begin{array}{l}\text { PÃES E } \\
\text { MASSAS }\end{array}$ & & & & & \\
\hline CEREAIS & & & & & \\
\hline CARNES & & & & & \\
\hline $\begin{array}{l}\text { LEITE E } \\
\text { DERIVADOS }\end{array}$ & & & & & \\
\hline OVOS & & & & & \\
\hline LEGUMINOSAS & & & & & \\
\hline VERDURAS & & & & & \\
\hline LEGUMES & & & & & \\
\hline $\begin{array}{l}\text { TUBÉRCULOS } \\
\text { E RAÍZES }\end{array}$ & & & & & \\
\hline FRUTAS & & & & & \\
\hline $\begin{array}{l}\text { ÓLEOS E } \\
\text { GORDURAS }\end{array}$ & & & & & \\
\hline $\begin{array}{l}\text { DOCES E } \\
\text { AÇÚCAR }\end{array}$ & & & & & \\
\hline
\end{tabular}

\section{DIAGNÓSTICOS CLÍNICOS:}

DIABETES ( ) HIPERTENSÃO ( ) DISLIPIDEMIA ( ) HIPERTRIGLICERIDEMIA ( ) HIPERCOLESTEROLEMIA ( ) CARDIOPATIA ( ) OUTROS: 
Análise da presença de síndrome metabólica em idosos atendidos no Projeto Atendimento Multidisciplinar ao Idoso (AMI) em Campo Grande, MS

\begin{tabular}{|c|c|c|c|}
\hline \multicolumn{4}{|c|}{$\begin{array}{l}\text { SE SIM, ASSINALE ABAIXO OS ANALISADOS: } \\
\text { METFORMINA ( ) NIMESULINA（） CAPTOPRIL（） LOSARTANA（） HIDROCLOROTIAZIDA（） SINVASTATINA（） } \\
\text { ASPIRINA（）OUTROS? }\end{array}$} \\
\hline \multirow{2}{*}{\multicolumn{4}{|c|}{ 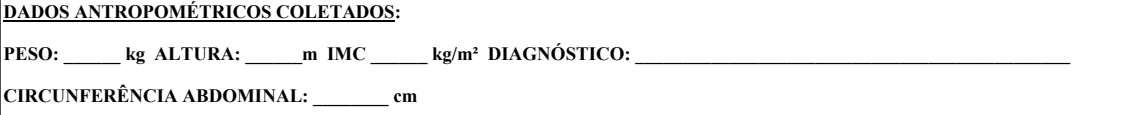 }} \\
\hline & & & \\
\hline \multicolumn{4}{|l|}{ EXAMES LABORATORIAIS: } \\
\hline & Valores & & Valores \\
\hline GLICEMIA $70-99$ mg/dL & & $\begin{array}{l}\text { LDL Ótimo: }<100 \mathrm{mg} / \mathrm{dL} \\
\text { Desejável: } 100-129 \mathrm{mg} / \mathrm{dL}\end{array}$ & \\
\hline $\begin{array}{ll} & \text { COLESTEROL TOTAL }<200 \\
\mathrm{mg} / \mathrm{dL} & \end{array}$ & & $\begin{array}{l}\text { COLESTEROL VLDL } 10 \text { a } \\
50 \mathrm{mg} / \mathrm{dL}\end{array}$ & \\
\hline HDL $>$ OU $=\mathrm{a} 40 \mathrm{mg} / \mathrm{dL}$ & & $\begin{array}{lll} & \text { TRIGLICERIDES } & <150 \\
\mathrm{mg} / \mathrm{dl} & & \end{array}$ & \\
\hline
\end{tabular}

***Formulário de pesquisa desenvolvido pelas pesquisadoras. 
